\title{
LA QUESTIONE DELLA COMPATIBILITÀ DELLE NORME \\ DELL'ORGANIZZAZIONE MONDIALE DEL COMMERCIO CON \\ QUELLE DEGLI ACCORDI PREFERENZIALI DI COMMERCIO E DELLE \\ SOVRAPPOSIZIONI DI GIURISDIZIONE TRA I LORO SISTEMI DI SOLUZIONE DI CONTROVERSIE*
}

THE FEASIBILITY OF THE WORLD TRADE ORGANIZATION RULES WITH PREFERENTIAL TRADE AGREEMENTS AND JURISDICTION OVERLAPS BETWEEN THESE DISPUTE SETTLEMENT

SYSTEMS

Umberto Celli Junior ${ }^{* *}$

1. Introduzione

Il diritto internazionale ha subito, soprattutto negli ultimi due decenni, un accentuato processo di frammentazione, responsabile per la comparsa di numerosi regimi normativi, molti dei quali incompatibili tra di loro. Tali regimi hanno pubblicato regole che hanno cominciato a controllare le più diverse aree della scena internazionale, quali quelle relative alla protezione dell'ambiente, alla lotta contro i cambiamenti climatici, alla protezione dei diritti umani, all'uguaglianza sociale e alla lotta contro la povertà, al commercio multilaterale e accordi commerciali regionali e bilaterali, tra molte altre. La mancanza di coordinamento tra i regimi normativi ha contribuito all'aumento della probabilità di conflitti tra di essi.

Questo potenziale di conflitti normativi è osservato con molta chiarezza nell'ambito del commercio internazionale, opponendo le regole del sistema multilaterale dell'Organizzazione Mondiale del Commercio ("OMC") alle regole degli Accordi Preferenziali Regionali e Bilaterali ("APC"). Malgrado gli APC riproducano regole basiche della OMC (OMC intra), essi hanno prodotto norme che vanno molto oltre a ciò che si trova attualmente regolamentato da quell'organizzazzione internazionale in aree quali commercio di servizi, proprietà intellettuale e misure di investimento relative al commercio (OMCplus), oltre a nuove regole per aree non ancora regolamentate dalla OMC,

\footnotetext{
Conferenza proferita nella Facoltà di Giurisprudenza dell' Università di Padova, Italia, 27/10/2011.L'autore è grato dell'amàbile invito che gli è stato fatto dal Professore Francisco Leita (Università di Padova) e delle osservazioni inestimàbili del Professore Alberto do Amaral Júnior (USP).

Questo artícolo è dedicato al mio padre, Umberto Celli, un gran uomo che da più da 50 anni lasciò la piccola e bella Tortoreto Alto, Provincia di Teramo, Abbruzzo, Italia, per costruire la sua vita nella nostra maestosa San Paolo.

** Professore di Diritto Internazionale della Facoltà di Diritto dell’Università di São Paulo (USP), Brasile.
} 
quali investimento, concorrenza, ambiente e standard lavorativi, tra l'altro (OMCextra). L'esistenza di questi numerosi APC - circa trecento in vigore - ha trasformato il commercio internazionale in un intricato sistema formato da sottosistemi sovrapposti, che si è convenzionato di denominare di spaghetti bowl. Notare che questa sovrapposizione non avviene soltanto tra gli APC in relazione all'OMC, ma anche tra gli APC, poiché vi sono paesi che fanno parte di più di dieci APC allo stesso tempo, come, per esempio, gli USA.

Questi APC, oltre a creare norme potenzialmente conflittuali e incompatibili con la struttura normativa della OMC, contengono sistemi propri di soluzione di controversie, generando la possibilità di conflitti di giurisdizione tra tali sistemi e l'Organo di Risoluzione delle Controversie ("OSC") della OMC. Si verificano, in genere, come ben sapete, tre tipi di conflitti di giurisdizione: i) quando due fori considerano di avere giurisdizione esclusiva sugli stessi argomenti o su argomenti paralleli o similari; ii) quando un foro rivendica di avere giurisdizione esclusiva su determinata materia mentre l'altra parte accetta di verificare i fatti senza postulare esclusività; c) quando due giurisdizioni se dispongono, volontariamente, a esaminare lo stesso litigio o litigi similari. Se non vi sono chiare indicazioni di come risolvere i potenziali conflitti dei sistemi di risoluzione delle controversie degli APC ("MSC") paralleli alla OMC, si troveranno in rischio l'autorità e l'effettività del sistema di governance internazionale del commercio.

In ogni modo, gli APC continueranno ad esistere e ad espandersi, fatto riconosciuto dalla $\mathrm{OMC}$, in un recente rapporto intitolato WTO Trade Report - 2011 - The WTO Preferential Trade Agreements: from co-existence to coherence, nel quale si afferma che "non c'è ragione per presumere che il numero di APC cesserà di crescere o che essi non faranno parte dello scenario delle relazioni commerciali internazionali".

Ciò che intendo discutere con voi, pertanto, è come la $\mathrm{OMC}$ e, specialmente, il suo OSC, hanno operato con questa realtà ed esplorare alcune possibilità di una coesistenza meno potenzialmente conflittuale tra le sue norme e quelle degli APC, oltre che tra i rispettivi sistemi di risoluzione di controversie. Con base nella giurisprudenza della $\mathrm{OMC}$, darò più enfasi ai casi che permettono riflessioni sui conflitti o sovrapposizioni (come preferisco chiamarli) di giurisdizione tra l'OSC e alcuni MSC di APC.

2. La questione della compatibilità tra le regole della $\mathrm{OMC}$ e quelle degli APC

Gli APC hanno il loro fondamento legale negli Articoli XXIV del GATT e V dell'Accordo Generale sul Commercio di Servizi (GATS) e nella Clausola di Abilitazione (APC tra paesi in via di sviluppo). Secondo le regole della OMC, la creazione degli APC per mezzo di Trattati Internazionali deve essere notificata al Comitato sugli Accordi Regionali ("CAR"). Il CAR ha la prerogativa di elaborare rapporti e raccomandazioni alle parti degli APC. In tal modo, ci sarebbe la possibilità da parte del CAR di riconoscere 
inconsistenze delle regole degli APC con il sistema multilaterale di commercio, caso non formassero un'unione doganale o una zona di commercio libero, nei termini dell'Articolo XXIV del GATT, oppure se violassero le disposizioni dell'Articolo V del GATS e della Clausola di Abilitazione.

Con il passar del tempo, quello che è avvenuto è che non è mai stato fatto un esame dettagliato, approfondito e conclusivo della compatibilità di un APC con la $\mathrm{OMC}$, per mancanza di volontà politica degli stessi Membri dell'OMC e per la velocità vertiginosa con cui gli APC hanno proliferato. A rigore, si è sempre saputo che nessun $\mathrm{APC}$ avrebbe superato il test di compatibilità.

Considerata l'inoperosità del CAR, nel 2006, il Consiglio Generale dell'OMC ha creato il cosiddetto Meccanismo di Trasparenza, un meccanismo provvisorio che si limita ad amministrare l'esistenza degli APC. Si tratta di un complesso di regole che esigono il previo annuncio e la notifica della firma degli APC alla OMC. Per mezzo di questo meccanismo, l'OMC passa a concentrarsi sulla trasparenza dei molteplici APC, senza peraltro sollevare questioni problematiche o critiche.

In tal modo, l'OSC rimarrebbe come forum di analisi dell'incompatibilità degli APC con il sistema multilaterale di commercio. La giurisprudenza dell'OMC dimostra che tale compatibilità finisce per essere valutata solo come una questione preliminare, quando una norma regionale viene contestata e l'eccezione dell'Articolo XXIV del GATT viene invocata. I panels e l'Organo d'Appello (“OA") dell'OMC evitano di decidere sul merito della compatibilità degli APC.

Il caso Turkey-Textiles costituisce il contenzioso in cui è stata affrontata più da vicino la questione della compatibilità tra le regole degli APC e le regole dell'OMC. Questo caso riguarda un'azione interposta di fronte all'OSC dall'India contro la Turchia. La Turchia aveva stabilito restrizioni quantitative ai prodotti tessili dell'India, allegando che erano necessarie per compiere i suoi obblighi con l'allora Comunità Europea ("CE”) nell'ambito dell'Accordo di Unione Doganale esistente tra entrambe. Secondo la Turchia, dato che la CE manteneva le proprie quote contro prodotti tessili indiani, l'imposizione di quote contro l'India era necessaria in forza del paragrafo $8^{\circ}$ dell'Articolo XXIV del GATT, secondo i cui termini i membri di un'Unione Doganale devono applicare sostanzialmente le stesse tariffe e altre regole nelle relazioni commerciali con paesi terzi. Il panel ha considerato che queste quote non erano incluse nell'Accordo sui Tessili dell'OMC e, pertanto, erano inconsistenti con l'Articolo XI del GATT (Eliminazione di Restrizioni Quantitative). Inoltre, d'accordo con il panel, l'Articolo XXIV del GATT non potrebbe mai essere utilizzato per convalidare una eccezione ad una restrizione di una misura quantitativa illegale. L'OA ha riformulato la raccomandazione del panel risaltando che, in principio, l'Articolo XXIV potrebbe essere utilizzato per convalidare qualunque violazione di un Articolo del GATT, purché fossero osservate due condizioni: (i) che la misura in questione fosse introdotta durante la formazione di una Unione Doganale che 
fosse in piena conformità con l'Articolo XXIV del GATT; e (ii) che la parte interessata dovrà dimostrare che la formazione dell'Unione Doganale non sarebbe possibile se non fosse stata autorizzata l'introduzione della misura in questione. In realtà, l'OA ha solo presunto la compatibilità con l'Articolo XXIV, senza effettuare analisi più profonde.

Quanto al MERCOSUL (Mercato Comune del Sud), il primo caso coinvolgendo potenziale conflitto tra le sue norme e quelle dell'OMC è stato Argentina Safeguard Measures on Imports of Footwear. Nel 1998, la CE ha questionato la legalità di misure di salvaguardia definitive imposte dall'Argentina contro l'importazione di calze da tutti i Membri dell'OMC, eccetto dai membri del MERCOSUL. L'Argentina ha allegato che, secondo l'Articolo XXIV del GATT e l'Accordo sulle Salvaguardie dell'OMC, essa era autorizzata ad escludere i membri del MERCOSUL da tali restrizioni considerando $\mathrm{i}$ suoi obblighi verso l'Unione Doganale (MERCOSUL).

Però la CE ha argomentato che, quando l'investigazione (per l'applicazione di salvaguardie) fatta dall'Argentina è stata realizzata, aveva preso in considerazione anche le importazioni dal MERCOSUR e che l'Argentina aveva deciso di escludere quelle importazioni dall'applicazione delle salvaguardie solo più tardi. L'OSC (panel e OA) si è limitato ad analizzare specificamente gli argomenti relativi all'Accordo Sulle Salvaguardie della OMC, avendo evitato l'esame della compatibilità del MERCOSUL con l'Articolo XXIV e la Clausola di Abilitazione. Secondo 1'OA, con base nell'Accordo di Salvaguardia, una misura di salvaguardia deve essere applicata sull'importazione di "tutte" le fonti di importazione considerate nell'investigazione per l'adozione delle citate misure e, per questo motivo, l'investigazione realizzata dalle autorità argentine era inconsistente con l'Accordo sulle Salvaguardie dell'OMC, dato che escludeva le importazioni dal MERCOSUL dall'applicazione della sua misura di salvaguardia, malgrado abbia incluso queste importazioni dal MERCOSUL durante l'investigazione.

Anche nel caso Brazil- Measures Affecting Imports of Retreated Tyres, la OSC ha avuto l'opportunità di esaminare il compimento delle norme del MERCOSUL con le regole del sistema della OMC per gli APC e, di nuovo, non ha voluto farlo. Come dirò più avanti, il caso, iniziato dalla $\mathrm{CE}$ contro il Brasile, ha coinvolto argomenti di natura ambientale e di salute pubblica, combinati con integrazione regionale. La CE ha messo in questione la legalità della misura adottata dal Brasile che proibiva l'importazione di pneumatici rinnovati da tutti i paesi, inclusi quelli della $\mathrm{CE}$, fatta eccezione per quelli del MERCOSUL. Alla fine, l'OSC ha condannato la misura brasiliana invocando la rationale del caso Turkey-Textiles, malgrado non abbia basato la sua decisione nell'Articolo XXIV. L'OA ha sostenuto che la rationale dell'Art. XX del GATT (eccezioni generali per permettere, tra l'altro, la protezione della vita umana, animale e vegetale o la salute) era necessaria al commercio internazionale, ma, secondo il doppio test stabilito dal caso Turkey-Textiles, la misura specifica non potrebbe costituire una ingiustificata o arbitraria discriminazione tra paesi con le stesse condizioni. 
Altri casi hanno avuto posizioni similari dall'OSC. Ciò che si verifica, pertanto, è che, perlomeno fino al momento presente, non esiste nessuna disposizione dell'OSC a realizzare un esame effettivo della compatibilità tra le norme dell'OMC e quelle degli APC. Come ho affermato anteriormente, è poco probabile che questo avvenga, poiché nessun APC sarebbe strettamente in conformità con le norme del sistema multilaterale.

Si tratta di una situazione complicata anche perché, alla ricerca di posizioni equilibrate, l'OSC tenta sempre di prendere in considerazione nei suoi processi le regole basiche del diritto internazionale e di interpretazione dei trattati. La prima di queste regole, come si sa, è che non esiste gerarchia inerente alle norme nel diritto internazionale. Tutti i trattati hanno lo stesso livello gerarchico, a meno che entrino in conflitto con una norma imperativa di diritto internazionale generale (jus cogens), caso in cui sono considerati nulli (Articolo 53 della Convenzione di Vienna sul Diritto dei Trattati). In tal modo, per esempio, il fatto che la OMC sia un trattato multilaterale e che una zona di commercio libero sia un trattato bilaterale non significa, per definizione, che la OMC debba essere superiore a questo o ad altri accordi commerciali. Tanto la OMC quanto il MERCOSUL sono dei trattati e, come tali, essi hanno, in principio, lo stesso valore.

Ma ci sono eccezioni. La prima di queste è quando disposizioni specifiche di un trattato lo mettono in posizione superiore ad un altro. Un trattato può stabilire che, in caso di conflitto, questo dovrà sottomettersi o prevalere su di un altro. L'Articolo 103.2 del NAFTA (Zona di Libero Commercio dell'America del Nord) prevede, per esempio, che, a meno che diversamente stabilito, il NAFTA dovrà prevalere sul GATT/OMC. Inversamente, l'Articolo 1.2.2. del Trattato di Libero Commercio tra gli Stati Uniti e la Corea stabilisce che la zona di commercio libero sarà sottomessa a qualunque obbligo legale internazionale tra le parti che preveda un trattamento più favorevole di quello previsto nel trattato tra entrambi i paesi (Articolo 41,1, a, della Convenzione di Vienna sul Diritto dei Trattati).

Un trattato multilaterale, d'altra parte, può prescrivere che un accordo bilaterale o regionale delle parti sia, in determinate circostanze, proibito (Articolo 41, 1, b, Convenzione di Vienna sul Diritto dei Trattati). È esattamente quello che fa l'Articolo XXIV quando proibisce la celebrazione di Unioni Doganali e Zone di Commercio Libero discriminatorie e che non osservano le condizioni in esso stabilite. Però, anche se questi trattati costitutivi "illegali” di Unioni Doganali o Zone di Commercio Libero sono firmati, il diritto internazionale presume che siano "legali" (cioè, compatibili con l'Articolo XXIV del GATT, Articolo V del GATS e Clausola di Abilitazione), a meno che l'OSC li dichiari "illegali" (incompatibili). Come ho specificato anteriormente, di fronte all'assenza di volontà politica o di decisioni dell'OSC quanto alla compatibilità degli APC 
con l'Articolo XXIV, tutti gli APC sono, pertanto, presunti come compatibili con l'OMC e continueranno ad operare, pur non essendolo in realtà.

L'Articolo 41.1, b, (i) della Convenzione di Vienna permette che solo due o più parti in un trattato multilaterale concludano un accordo per modificare il trattato, soltanto tra le stesse, purché questo non pregiudichi il godimento per le altre parti dei diritti provenienti dal trattato e il compimento dei loro obblighi. Per questa ragione, nell'opinione dell'OSC, solo le regole dell'OMC, quali l'Articolo XXIV o XX, potrebbero giustificare la violazione, da parte del Brasile, del Trattato dell'OMC e non quelle del MERCOSUL.

E per questo che molti autori, tra i quali mi includo, sostengono che la realtà politica e legale è indicativa del fatto che gli APC continueranno ad esistere e ad espandersi, indipendentemente dalla loro compatibilità con le regole dell'OMC. Così, invece di lamentare l'incompatibilità o restare esasperati con lo "spaghetti bowl" risultante, forse sarebbe più produttivo pensare come questa rete di APC può interagire $\mathrm{e}$ dialogare con le norme dell'OMC, in modo da assicurare maggior efficacia per entrambi.

3. Riflessioni sulle sovrapposizioni di giurisdizione tra l'OSC della OMC e gli MSC degli APC

L'Articolo 23.1. dell'Intesa Sulle Norme e Procedure che disciplinano la Risoluzione delle Controversie dell'OMC (Dispute Settlement Understanding- DSU) ha conferito all'OSC giurisdizione obbligatoria ed esclusiva per conoscere e giudicare violazioni agli Accordi dell'OMC. Un Membro dell'OMC ha il dovere di ricorrere e accettare le norme ed i procedimenti del DSU per risolvere qualunque violazione degli Accordi dell'OMC. Dopo che si è azionato l'OSC, la giurisdizione di qualunque altro meccanismo è automaticamente esclusa.

Anche se il sistema di risoluzione delle controversie di un APC rivendica giurisdizione esclusiva su un determinato tema (l'Articolo 2005 del NAFTA, per esempio, stabilisce giurisdizione esclusiva del suo MSC nei casi relativi alla protezione dell'ambiente e applicazione di misure sanitarie e fitosanitarie adottate da una delle parti per proteggere la vita o la salute umana, animale e vegetale), se una misura (norma o decisione arbitrale) adottata nel suo ambito implica violazioni degli Accordi dell'OMC, l'OSC potrà essere azionato parallelamente. Nel caso US-Wool Shirts and Blouses, 1'OA dell'OSC ha già avuto l'opportunità di assumere una posizione a riguardo quando ha deciso che qualunque Membro dell'OMC, che sia anche parte integrante di un APC e che consideri che i suoi diritti sotto l'OMC siano stati in qualche modo limitati, potrà azionare l'OSC dell'OMC.

Niente impedisce un Membro dell'OMC di azionare, indistintamente, l'OSC dell'OMC o l'MSC di un APC, considerando l'assenza di norme internazionali che regolino la relazione tra i procedimenti di soluzione di dispute. 
La sovrapposizione giurisdizionale può avvenire, allora, quando esiste una violazione passibile di azione in entrambi i fori. Preferisco utilizzare il termine sovrapposizione, poiché, in principio, non dovrebbe esserci conflitto di giurisdizione, dato che l'OSC applica le norme dell'OMC, mentre gli APC applicano le loro rispettive norme.

Nel caso Caso Mexico - Tax Measures on Soft Drinks and Other Beverages, l'OSC ha deciso che il DSU non potrebbe essere usato per applicare, rinforzare o affermare diritti ed obblighi esterni ai trattati dell'OMC.

Come risposta alla lentezza degli Stati Uniti nell'impiantare una misura accordata nell'ambito del NAFTA che ridurrebbe le tasse nordamericane di importazione dello zucchero messicano e davanti al fallimento dei tentativi di instaurare un panel in ambito regionale, il Messico ha ridotto l'importazione di bevande nordamericane che non fossero state addolcite con zucchero derivato da canna.

Gli Stati Uniti, allora, hanno azionato l'OMC, affermando che la misura messicana era un affronto alle regole del sistema multilaterale di commercio. Pur con la richiesta del Messico affinché 1'OSC dell'OMC declinasse la sua giurisdizione, allegando che la controversia avrebbe dovuto essere risolta da un panel del NAFTA, la decisione è stata favorevole agli Stati Uniti ed ha aperto un precedente nel senso che il DSU non può essere usato per applicare, rinforzare o affermare diritti ed obblighi esterni agli accordi dell'OMC. In tal modo, tutte le allegazioni messicane basate in dispositivi del NAFTA sono state rifiutate dal panel e dall'OA.

Malgrado non ci sia stato propriamente un conflitto di giurisdizioni tra il meccanismo di soluzione di controversie del NAFTA e l'OSC dell'OMC, il precedente ha aperto la strada per la sovrapposizione di decisioni in ambito regionale e multilaterale.

Nel caso Canada - Certain Measures Concerning Periodicals riguardante una controversia tra Stati Uniti e Canada sulle tassazioni adottate da quest'ultimo paese su edizioni split-run della rivista nordamericana Sports Illustrated, tanto il panel quanto l'OA hanno deciso a favore degli Stati Uniti. Tuttavia, la tassazione canadese era stata effettuata nei termini di quanto accordato dalle parti nel NAFTA, che esime le industrie culturali dal vincolo dell'accordo.

In altre parole, se la controversia fosse stata presentata ad un panel del NAFTA, la decisione arbitrale sarebbe stata probabilmente favorevole al Canada, dato che la legge applicabile prevedeva espressamente la possibilità di tassazione che proteggesse industrie culturali domestiche come quelle di riviste. Siccome la disputa è stata nell'ambito dell'OMC, gli obblighi regionali non sono stati esaminati. L'OSC ha solo preso in considerazione il fatto che il Canada non ha ubbidito le regole del sistema multilaterale di commercio.

In entrambi i casi, vale notare che l'OSC ha applicato esclusivamente le regole del sistema multilaterale e il conflitto di giurisdizione non si è materializzato, perché i panels nell'ambito del NAFTA non sono stati effettivamente instaurati (né prima, 
né dopo la decisione in ambito multilaterale). Però hanno aperto precedenti rilevanti - soprattutto il caso Mexico - Soft Drinks - riguardo il parere dell'OSC su eventuali sovrapposizioni di giurisdizione. Ossia, nonostante non si sia materializzato il conflitto di giurisdizione, si deduce che, se il Messico e il Canada fossero riusciti ad instaurare un panel nell'ambito del NAFTA, le decisioni verrebbero ignorate dall'OMC, il cui panel e OA probabilmente terrebbero conto soltanto degli obblighi firmati in ambito multilaterale.

Invece il caso Brazil - Measures Affecting Imports of Reatreated Tyres ha configurato propriamente un conflitto di giurisdizione.

Il Brasile aveva proibito l'importazione di pneumatici rinnovati con l'argomento che il materiale era dannoso all'ambiente. Il caso è stato portato inizialmente dall'Uruguay al Tribunale Arbitrale del MERCOSUL, secondo le procedure del Protocollo di Brasilia, allora vigente. Nella disputa, il Brasile non ha utilizzato l'argomento ambientale (previsto nell'Associazione Latino Americana di Integrazione - ALADI, di cui il MERCOSUL fa parte) e la proibizione è stata considerata incompatibile con le norme di libero commercio del MERCOSUL. La decisione del Tribunale Arbitrale ha determinato che il Brasile doveva ritirare la proibizione ai paesi del MERCOSUL, ma ha mantenuto la proibizione ai pneumatici rinnovati importati da altri paesi. Conseguentemente, il Brasile, in obbedienza alla decisione del Tribunale Arbitrale del MERCOSUL, è tornato ad importare i pneumatici rinnovati dai membri del MERCOSUL, soprattutto dall'Uruguay.

La CE ha portato tale decisione del Brasile all'OSC. L'OA ha riconosciuto la compatibilità della misura con l'eccezione prevista nel capoverso (b) dell'Articolo XX del GATT sostenuta dal Brasile, ma ha accettato l'argomento della CE che era discriminatoria la distinzione fatta tra i membri del MERCOSUL e i Membri dell'OMC, nei termini del caput dell'Articolo XX del GATT, non riconoscendo la decisione arbitrale del MERCOSUL con l'allegazione che non aveva alcuna relazione con la protezione intesa dall'Articolo XX (b). Ha determinato, infine, che il Brasile proibisse l'importazione di pneumatici rinnovati da tutti i membri del MERCOSUL.

Nel caso Argentina Poultry, il governo argentino aveva adottato soprattasse (diritti antidumping) all'importazione di polli dal Brasile sotto il fondamento che gli esportatori brasiliani di pollame stavano vendendo il prodotto a prezzi inferiori a quelli praticati all'origine (dumping).

Chiamata di fronte al Tribunale Arbitrale del MERCOSUL, l'Argentina ha ottenuto parere favorevole, ossia, il Tribunale ha considerato che effettivamente esisteva la pratica di dumping. Non accettando la decisione del Tribunale Arbitrale, il Brasile ha portato la controversia all'OSC dell'OMC, allegando che le misure adottate dall' Argentina violavano le regole del sistema multilaterale di commercio.

Malgrado 1'argomentazione dell'Argentina che il caso non avrebbe potuto essere valutato dall'OMC, dato che il Tribunale Arbitrale del MERCOSUL aveva già emesso parere a riguardo, la controversia è stata analizzata dal panel dell'OMC, il quale 
ha considerato le soprattasse inconsistenti con l'Accordo Antidumping dell'OMC, determinando, pertanto, la loro ritirata. L'Argentina non è riuscita a comprovare la pratica di dumping da parte degli esportatori brasiliani, né il danno ai produttori argentini.

Queste sovrapposizioni di giurisdizione sono state oggetto di diverse analisi. Buona parte di loro tenta di trovare soluzioni in alcuni principi di diritto internazionale generale, quali, (i) forum conveniens e forum non conveniens; (ii) res judicata; e (iii) judicial comity. Tuttavia, questi principi sono molto specifici o non sono adattabili alla relazione tra l'OMC e gli APC. Il principio forum conveniens, secondo il quale un foro si dichiara appropriato in detrimento di un altro, non sarebbe applicabile, visto che, secondo l'Articolo 23 del DSU, l'OSC dell'OMC si dichiarerà sempre come foro appropriato. La res judicata, come sapete, implica il rifiuto della giurisdizione soltanto quando la causa dell'azione è la stessa nelle due istanze di litigio. Siccome le azioni si basano nella violazione di accordi differenti, l'applicazione del principio non sarebbe una soluzione. Il judicial comity è il principio che permette a un tribunale di declinare l'esercizio di giurisdizione su argomenti che potrebbero essere risolti in modo più adeguato in un altro tribunale. Tale principio si scontra con le disposizioni dell'Articolo 3.2 del DSU, secondo le quali le raccomandazioni e decisioni dell'OSC non potranno promuovere l'aumento o la diminuzione dei diritti e obblighi stabiliti negli accordi dell'OMC.

Gli specialisti nell'area stanno discutendo altre proposte che partono dalla premessa a cui mi sono riferito anteriormente che debba avvenire mutuo riconoscimento, accomodazione e rispetto tra l'OSC e gli MSC degli APC.

Per quanto riguarda l'OMC, vi sono suggerimenti nel senso che l'OSC dovrebbe rispettare le clausole di esclusione di foro o di giurisdizione esclusiva degli APC. A questo scopo, dovrebbero essere effettuate alterazioni al DSU, il che, considerato il sistema di decisione consensuale dell'OMC, è poco probabile che accada attualmente.

In relazione agli APC, segnalo le seguenti proposte: (i) i loro sistemi di risoluzione delle controversie dovrebbero stabilire che i panels arbitrali prendessero obbligatoriamente in considerazione le regole dell'OMC e la giurisprudenza dell'OSC; (ii) l'adozione di una "clausola di elezione di foro esclusivo" determinando che, dopo che la controversia sia stata sottoposta all'OSC o ad un panel di un APC, tale controversia non possa essere giudicata in un altro foro. È quanto già prevede, per esempio, 1'MSC del MERCOSUL (Protocollo di Olivos che sostituisce il Protocollo di Brasilia); (iii) quando gli obblighi degli APC fossero identici o sostanzialmente equivalenti a quelli già assunti dalle parti nell'OMC (OMCintra), gli MSCs degli APC dovrebbero dirigere la parte demandante a sottomettere la disputa all'OSC; e (iv) soltanto per quanto riguarda gli obblighi OMCplus e OMCextra si dovrebbero azionare automaticamente gli MSC.

Queste proposte non sono ovviamente consensuali, il che mi fa credere che siamo ancora molto lontani dal risolvere il problema delle sovrapposizioni di giurisdizione tra l'OSC dell'OMC e gli MSC degli APC. 


\section{Considerazioni finali}

Se, come ho affermato durante la mia esposizione, gli APC continueranno ad esistere e ad espandersi, la miglior cosa da fare, tanto per quanto riguarda la questione della compatibilità delle loro norme con quelle dell'OMC, quanto per quello che riguarda la sovrapposizione di giurisdizione dell'OSC con i suoi MSC, è cercare forme che permettano maggior interazione e dialogo tra i due sistemi. Il WTO Trade Report - 2011, all'accennare con la transizione dalla coesistenza verso la coerenza tra norme sostantive e procedurali, mi sembra un importante passo in questa direzione. 